\title{
Two new species of Meropidia Hippa \& Thompson, I 983 (Diptera, Syrphidae) from the Andes Mountains
}

\author{
Mírian N. Morales ${ }^{1, \dagger}$, Gunilla Ståhls ${ }^{1, \ddagger}$, Heikki Hippa ${ }^{2, \S}$ \\ I Finnish Museum of Natural History, PO Box 17, FI-00014 University of Helsinki, Finland 2 Gribbylunds \\ allé 2, SE-183 65 Täby, Sweden \\ † http://zoobank.org/EC0753BF-84A8-4609-9723-69AA461B5D2B \\ † http://zoobank.org/D19B1262-503A-48E2-91AE-F99D0F2EA3A3 \\ § http://zoobank.org/546524AD-3BD1-4830-842F-EEEE7DF4799B \\ Corresponding author: Mirian N. Morales (mirian_nm@yahoo.com.br)
}

Academic editor: Ximo Mengual | Received 12 August 2013 | Accepted 11 September 2013 | Published 2 October 2013

http://zoobank.org/E283E3F0-8623-4BAC-993F-008793A66674

Citation: Morales MN, Ståhls G, Hippa H (2013) Two new species of Meropidia Hippa \& Thompson, 1983 (Diptera, Syrphidae) from the Andes Mountains. ZooKeys 338: 55-65. doi: 10.3897/zookeys.338.6093

\section{Abstract}

Two new species of Meropidia Hippa \& Thompson, 1983 (Diptera, Syrphidae) are described, Meropidia nitida Morales, sp. n. and M. flavens Hippa \& Ståhls sp. n., from Bolivia and Colombia respectively. A key to all described Meropidia species is provided.

\section{Keywords}

Description, Eristalinae, Meropidia, identification key, Neotropical region, taxonomy

\section{Introduction}

The Neotropical region has a very high diversity of flower flies (Diptera, Syrphidae), comprising just over $30 \%$ of the currently recognized species in the world (Thompson et al. 2010) and many species new to science have continuously been described for genera confined to this region (e.g. Rotheray et al. 2007, 2009, Carvalho Filho and Esposito 2009, Morales et al. 2009, Ricarte et al. 2012). However, few studies on re- 
gional Syrphidae fauna in the Neotropical region exist, particularly on South America (see Thompson and Marinoni 2003, Montoya et al. 2012 for a review).

The genus Meropidia was described by Hippa and Thompson in 1983, comprising three species: Meropidia nigropilosa Thompson, M. neurostigma Hippa and M. rufa Thompson. All described species are confined to the Neotropical region, specifically occurring on Tropical Andes (Hippa and Thompson 1983, Montoya et al. 2012).

Meropidia Hippa \& Thompson, 1983 belongs to the subfamily Eristalinae and was placed in the tribe Milesiini, subtribe Tropidiina (Hippa and Thompson 1983). This subtribe comprises seven genera in addition to Meropidia: Calcaretropidia Keiser, 1971, Macrozelima Stackelberg, 1930, Nepenthosyrphus Meijere, 1932, Orthoprosopa Macquart, 1850, Senogaster Macquart, 1843, Syritta Lepeletier \& Serville, 1828 and Tropidia (Meigen, 1822). Of these genera only Meropidia and Senogaster are confined to the Neotropical region, while species of Syritta and Tropidia also occur in the region (Thompson 2013). These genera can be readily distinguished from Meropidia by a swollen metafemur, often with armature, as Meropidia has a slightly thickened throughout and slightly arcuate metafemur, with no modifications (Thompson 1972).

Besides the simple metafemur, Meropidia is recognized as a moderately pilose taxa, with sexually dimorphic face (male with a broad, low, medial tubercle and female concave), with pollinose pattern on mesonotum and pilose metasternum. It has eye bare and male is very narrowly dichoptic. The wing has variable numbers of stigmatic crossveins; cell $\mathrm{r} 1$ open; vein $\mathrm{R} 4+5$ slightly sinuate, with a short petiole; vein $\mathrm{r}-\mathrm{m}$ strongly oblique and long, ending at outer $1 / 4$ of discal cell, vein $\mathrm{A} 1+\mathrm{CuA} 2$ very long. The male sex is known only for one species, M. neurostigma (Hippa and Thompson 1983). The authors of this genus suggest that Meropidia is sister group of Orthoprosopa and Paratropidia Hull, 1949, being these three taxa the plesiomorphic sister group to the other genera of Tropidiina (Hippa and Thompson 1983).

The most appropriate identification key to run for the genus Meropidia is that published in Thompson (1999). Because Meropidia is a small genus with species confined to a very particular region, there is no information about biology of its species, neither about immature stages. In addition to the original description work (Hippa and Thompson 1983) and the identification key for Neotropical genera (Thompson 1999), Meropidia is also cited by Montoya et al. (2012), where is presented a review for flower flies fauna of Colombia and a comprehensive review of the literature concerning Colombia and the Neotropical region. It is noteworthy that in a previous work presenting a faunal list of Colombia, Gutierrez et al. (2005) had omitted the genus Meropidia from that list, but Montoya et al. (2012) included the omitted species Meropidia neurostigma Hippa, 1983 and recorded M. nigropilosa Thompson, 1983 as new to Colombia.

No additional species have been described for Meropidia since 1983, and in the present work two new species, Meropidia nitida Morales, sp. n. and M. flavens Hippa $\&$ Ståhls, sp. n., from Bolivia and Colombia respectively, are described. 


\section{Material and methods}

Terminology follows Thompson (1999). The identification key was constructed based on the key from Hippa and Thompson (1983).

Type localities and holotype holding institutions are specified for each species. Location and identifications labels are indicated with quotation marks (“”), and which line on the label separated by a forward slash (/). Handwritten information on labels is indicated in italics. The acronyms used for collections follow the standard of the Systema Dipterorum (Thompson 2013), and their equivalents are listed below:

AMNH American Museum of Natural History, New York, USA.

MNHN Muséum National d'Histoire Naturelle, Paris, France.

All measurements are in millimeters and were taken using a reticule in a Nikon SMZ1000 stereomicroscope. Photographs of all new species were provided and were composed using the Combine ZP software based on images of pinned specimens.

In addition, were also provided photographs of the $M$. nigropilosa holotype, which is deposited at Natural History Museum, London, UK, and has no images available in an online source.

\section{Description of new species}

The species described here do not have a concave face, neither a wholly pollinose face as defined in Hippa and Thompson 1983 as diagnostic characters for Meropidia females; but, it is flat with a sub-medial and sub-developed tubercle, and shiny medially. A full generic description of Meropidia is not necessary here, but it is necessary to record that concavity and pollinosity of female face are not diagnostic characters for the genus Meropidia. Otherwise we have no additions or changes to the generic description.

\section{Meropidia nitida Morales, sp. $\mathbf{n}$.}

http://zoobank.org/791B17B7-CC3C-4413-A750-8EDD936EBF7C http://species-id.net/wiki/Meropidia_nitida

Figs $1-8$

Type specimens. HOLOTYPE. Adult female, missing left midleg. Deposited at AMNH, New York City, USA. Type locality: BOLIVIA. Labels (Figs 6-8) "BOLIVIA: Sta Cruz Dept. / Caballero Prov., PN Amboró / 1750.124'S, 64²3.454'W / 2070 m, X.18-19.2001 / S. Spector \& J. Ledezma / mallaise sic' trap"; "COBIMI0011824"; "HOLOTYPE / Meropidia nitida / Morales, 2013". 

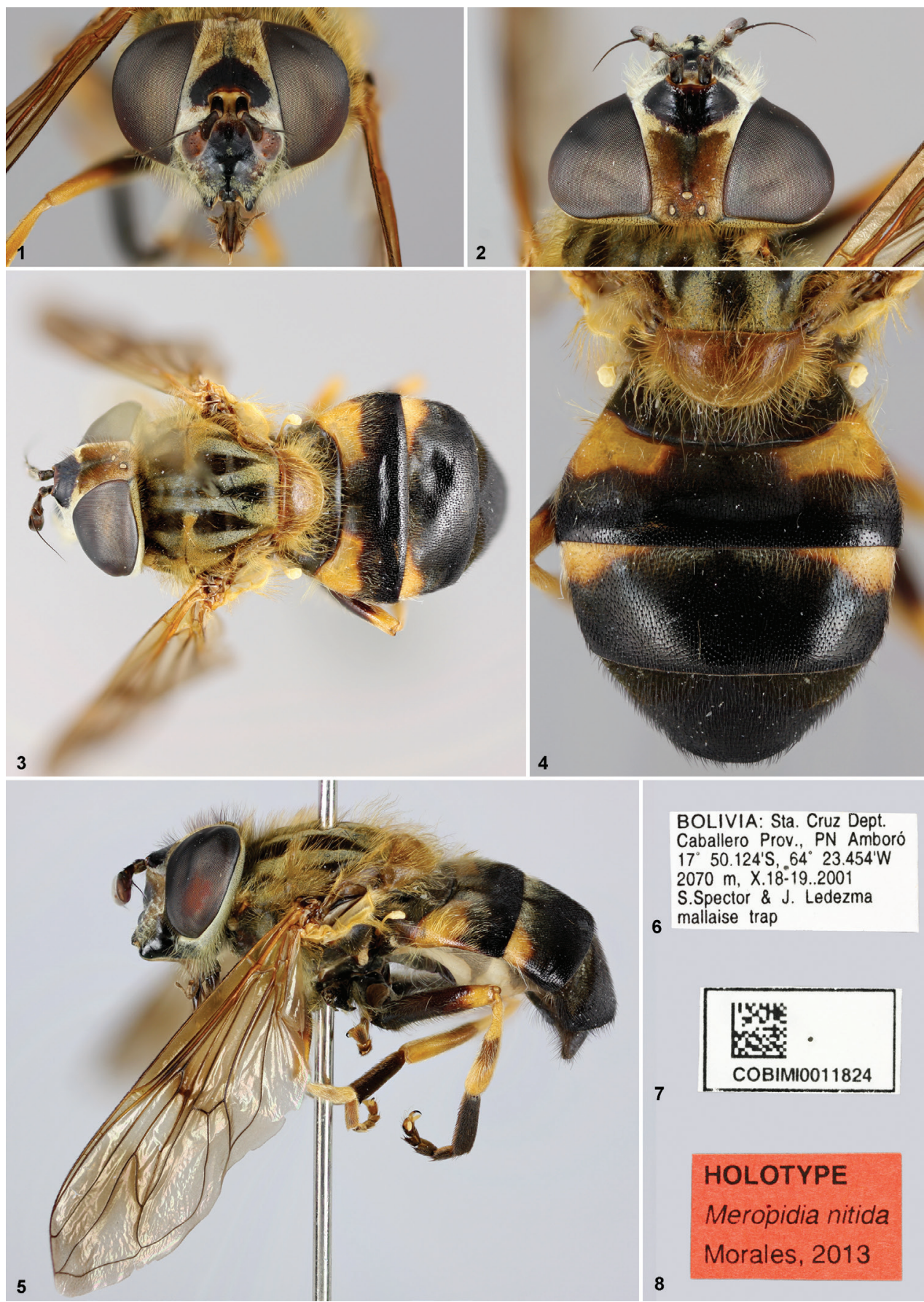

Figures I-8. Meropidia nitida Morales, sp. n., Holotype. I head, anterior view $\mathbf{2}$ head, dorsal view $\mathbf{3}$ habitus dorsal $\mathbf{4}$ abdomen, dorsal view $\mathbf{5}$ habitus lateral 6-8 labels. 
Paratype. Adult female. BOLIVIA. Deposited at AMNH, New York City, USA. Labels: same data as Holotype; "COBIMI0011826"; "PARATYPE / Meropidia nitida / Morales, 2013”.

Description. Adult female. Body size: 9-10.5 mm

Head. Face shiny black medially, becoming brownish to yellowish laterally, white pollinose laterally to bases of antenna, yellow pilose on pollinose area (Figs 1, 5), with a subdeveloped tubercle on inferior half (Fig. 5); gena shiny black, white pollinose and yellow pilose posteriorly (Fig. 5); facial stripes brown pollinose, with short yellow pile; lunula dark brown; frons shiny black in an anterior triangular area (Figs 1, 2), with brownish pile which became yellowish on their apexes, yellow pollinose elsewhere, sparse medially, intermixed with brownish and yellow pile; ocelli yellowish; ocellar triangle slightly isosceles obtuse (Fig. 2); occiput dark, yellowish pollinose dorsally, white pollinose elsewhere (Fig. 5), brownish and yellowish pilose dorsally, light yellow pilose elsewhere. Antenna. Scape and pedicel $2 \times$ longer than broad, dark brown (Fig. 1); arista dark brown, except apex reddish, with very short vestiture, downy; distance between antenna approximately 1.5 times its basal diameter (Figs 1, 2).

Thorax. Postpronotum yellow, densely pale pollinose and yellow pilose; scutum black, golden pollinose on lateral margins and on transverse suture, four longitudinal pale pollinose vittae extending in front of scutellum (Fig. 3), long yellow pilose (Fig. 5); scutellum yellowish, with longer yellow pile than scutum (Figs 3, 4), with scattered brownish pile on margin. Postalar callus with longer pile than scutum pile, as long as in scutellum, anterodorsal edge with long pile on posterior half, postalar wall bare. Calypter, haltere, plumula and spiracles yellow. Pleura dark brown, sparsely pale pollinose, except densely pollinose on posterior anepisternum, dorsal part of katepisternum and anterior anepimeron. Proepimerum, posterior anepisternum, anterior anepimeron and katatergum yellow pilose; pile of posterior anepisternum and anterior anepimeron somewhat produced posteriorly. Katepisternum mostly yellow pilose, except a few dark brown pile ventrally; metasternum black pilose.

Legs: simple, coxae and trochanters dark brown, mostly yellow pilose, intermixed with brownish pile. Pro and mesofemora brownish on basal half and yellow on apical half, yellow pilose, with longer pile ventrally; metafemur mostly dark brown, but apically yellow (Fig. 5), yellow pilose, ventral pile shorter than anterior and posterior surfaces, but longer than dorsal surface, longest pile on anterior and posterior surfaces erect, tilted apically elsewhere. Tibiae yellow, except metatibia slightly brownish medially (Fig. 5), yellow pilose. Metatarsus dark brown (Fig. 5), mostly brownish pilose, with few scattered yellow pile on anterior and posterior surfaces; pro and mesotarsus brown, lighter than metatarsus, yellow pilose.

Wing: completely microtrichose; vein $\mathrm{CuP}$ long, almost reaching the level of posterior apex of cell bm (Fig. 5); alula shorter than anal lobe;

Abdomen. Tergum I black (Figs 3, 4), lateral corners brownish yellow, with long yellow erect pile laterally, shortest black tilted backward pile elsewhere; tergum II mostly black, with yellow pile on the yellow maculae region (Figs 3, 4), laterally they are erect and longer, on anteromedial $1 / 3$ yellow pilosity tilted backward, pile black and 
tilted backward elsewhere, scattered pale pollinose on anterior half; tergum III mostly black, with yellow pile on lateral corners and on the yellow macula region (Figs 3, 4), black, posteriorly inclined pile elsewhere, whitish pollinose on anterior half; tergum IV black (Figs 3, 4), black pilose, except white pilose on anterior corners, whitish pollinosity forming two anterior triangular-like macula. Sterna dark brown, slightly pale pollinose; sterna I-III yellow pilose, IV yellow pilose intermixed with brownish pile; sternum $\mathrm{V}$ brownish pilose.

Comments. The holotype has four stigmatic crossveins on the left wing and five on the right; in the paratype there are six stigmatic crossveins on both wings. Only type material is known.

\section{Meropidia flavens Hippa \& Ståhls, sp. n. http://zoobank.org/5B54C6D3-4E88-4DD3-A585-810EB9DED488 http://species-id.net/wiki/Meropidia_flavens} Figs 9-15

Type specimen. HOLOTYPE. Adult female, in good condition, except arista missing. Postabdominal segments $\mathrm{V}$ and beyond removed and placed in plastic vial on the same pin as the specimen. Deposited at NMNH, Paris, France. Type locality: COLOMBIA. Labels (Figs 13-15) "Bogota / October / 1892"; "This lable 'sic'/ made by / Hippa I 1984"; "HOLOTYPE / Meropidia flavens / Hippa \& Ståhls, 2013”.

Description. Adult female. Body size: $11 \mathrm{~mm}$

Head. Elliptical in frontal view, (Fig. 11); face with a subdeveloped tubercle on inferior half (Fig. 10), shiny yellow medially, with brown U-shaped shiny area surrounding tubercle (Fig. 9), yellowish pollinose elsewhere, yellow pilose on pollinose areas; gena densely pale pollinose and long yellow pilose (Fig. 10); facial stripes brownish, short, matte, pale pollinose and pilose; lunula yellow, frontal area above lunula dark brown (Fig. 9), bare, brownish pilose; frons broad, densely yellowish pollinose and long pale pilose, antero-medial roundish and ocellar area bare; ocellar triangle slightly isosceles obtuse (Fig. 11), brownish long pilose; occiput densely pale pollinose, pale pilose, with some short black pile. Antenna: Scape brownish, $2 \times$ longer than broad, short dark pilose dorsally; pedicel brownish about 2.5-3× longer than broad, with short dark pile dorsally and ventrally; basoflagellomere darkbrown, shorter than high (Figs 9, 10); distance between antenna approximately twice its basal diameter (Fig. 9).

Thorax. Postpronotum yellow, densely pale pollinose and yellow pilose; scutum blackish, with four broad, longitudinal pale pollinose vittae extending to transverse pollinose area in front of scutellum (Fig. 11), long pale pilose, pale pollinose around transverse suture; anterodorsal edge of postalar callus with long pile on posterior half; scutellum yellowish (Fig. 11), long pale pilose, margin with pale pilosity with apex becoming browner, pile as long as scutellum. Calypters yellow with yellow marginal pilosity. Haltere yellow. Pleura brownish, slightly pollinose, except densely pale pollinose on posterior anepisternum, dorsal katepisternum and katepimeron. 

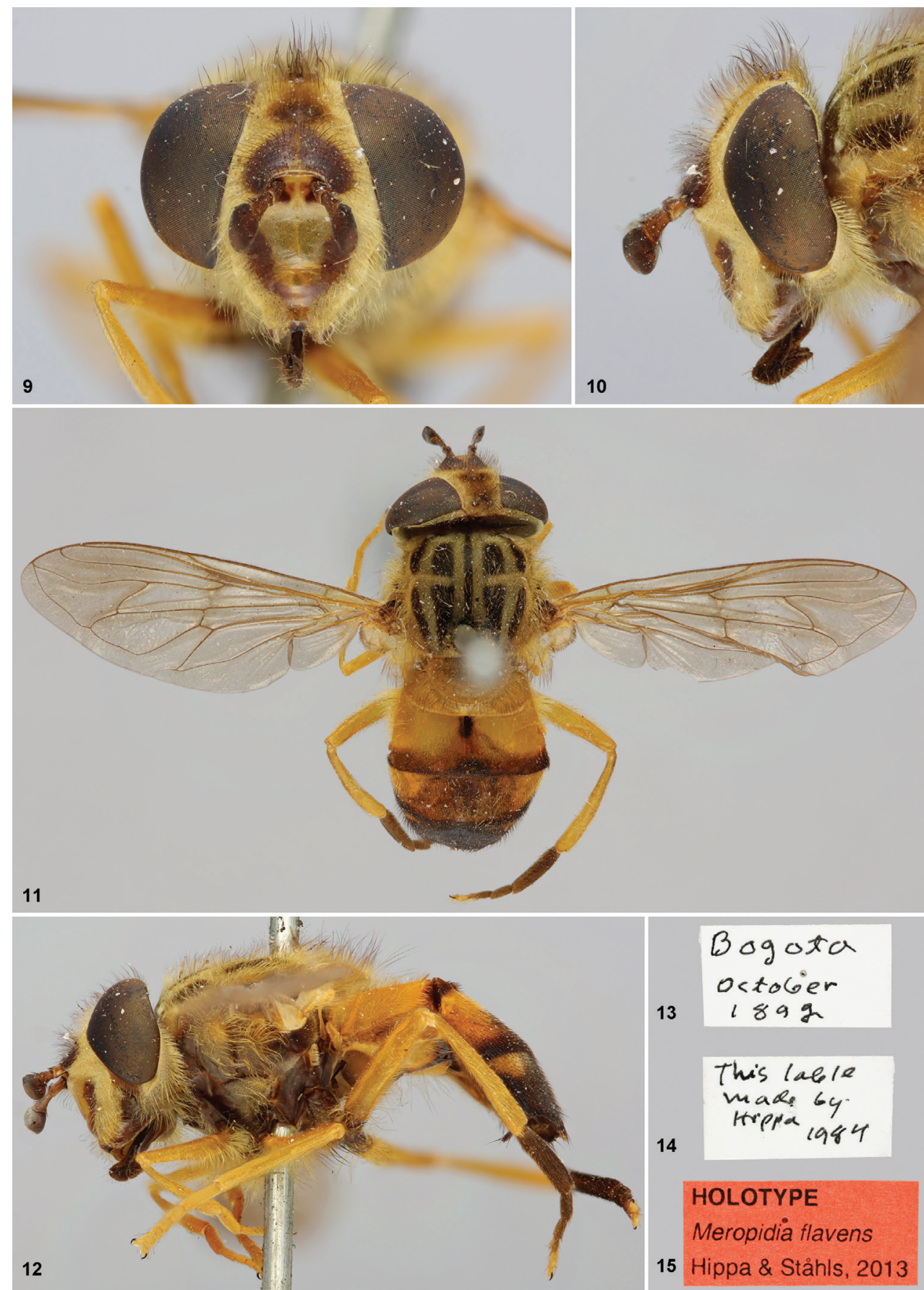

Figures 9-15. Meropidia flavens Hippa \& Ståhls, sp. n., Holotype 9 head, anterior view $\mathbf{l} 0$ head, lateral vie I I habitus dorsa $\mathbf{2}$ habitus lateral I 3-I 5 labels. 
Propleura, posterior anepisternum, dorsal and ventral katepisternum, anepimeron with long yellow pilosity, katatergum with shorter yellow pilosity; metasternum black pilose.

Legs: simple, metafemur straight; yellow, except for coxae and trochanters brown (Fig. 12), femora narrowly brown antero-basally, and metatarsal segments 1-4 dark brown (Figs 11, 12).

Wing: completely microtrichose; slightly brownish along veins, wing veins brownish-yellow; four stigmatic crossveins; vein CuP long, almost reaching the level of posterior apex of cell bm (Fig. 11); alula shorter than anal lobe.

Abdomen. Tergum I yellow (Fig. 11), yellow pilose anteriorly, black short pilose posteriorly; tergum II yellow with brown spot antero-medially and posterior brownish stripe (Fig. 11), with long yellow pile at anterior corners and triangular area with short black pile; tergum III with antero-lateral corners broadly yellow (Fig. 11) and with yellow pile, with posterior brownish stripe with short black pile; tergum IV brown with antero-lateral corners yellow (Fig. 12) with short black pile. Sterna brownish, long pale pilose; sternum II with lateral areas yellow.

Comments. Only type known.

\section{Identification key for the Meropidia species}

$1 \quad$ First tergum black (Figs 4, 16), at least yellowish to orange on posterior margin; metafemur mostly black, at least on basal $1 / 5$.................................... 2

- $\quad$ First tergum and femora entirely yellow or orange .................................... 4

$2 \quad$ Pro and mesofemora orange (Fig. 17); metafemur very narrowly black in basal 1/5 or less; metabasitarsus partially black (Fig. 17); mesonotum and scutellum with extensive black pile; (Colombia)

Meropidia nigropilosa Thompson

- $\quad$ Pro and mesofemora black on basal half and yellow on apical half; mesonotum and scutellum with extensive yellow pile....

3 Face shiny black medially (Fig. 1); frons shiny black in an anterior triangular area (Figs 1, 2); metatibia slightly brownish medially (Fig. 5); (Bolivia)

Meropidia nitida Morales, sp. $\mathbf{n}$.

- $\quad$ Face and frons (or frontal triangle) yellowish orange, extensively yellow pollinose; metatibia entirely yellow, without markings; (Bolivia)

Meropidia neurostigma Hippa

$4 \quad$ Face shiny yellow medially, with a brown U-shaped shiny area surrounding tubercle (Fig. 9); scutum with broad and longitudinal black markings (Fig. 11); metatarsal segments 1-4 dark brown (Figs 11, 12); (Colombia) ....

Meropidia flavens Hippa \& Ståhls, sp. n.

- $\quad$ Face orange, without dark brown markings surrounding tubercle; scutum with very narrow longitudinal black markings; metatarsal segments orange; (Ecuador) 


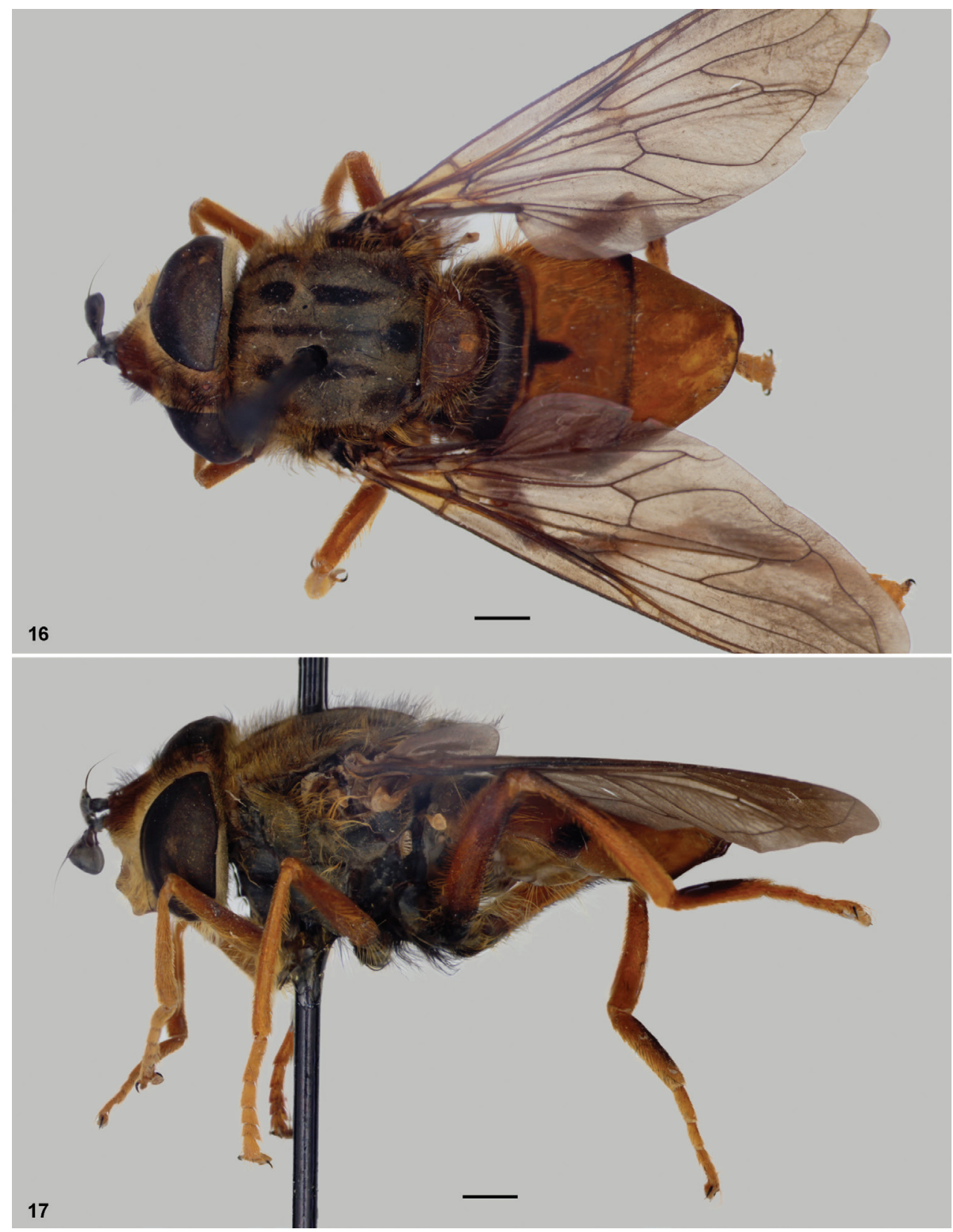

Figures 16-I7. Meropidia nigropilosa Thompson. Holotype deposited at Natural History Museum, London, UK $\mathbf{I} \mathbf{6}$ habitus dorsal $\mathbf{} 7$ habitus lateral. Scale bar: $1 \mathrm{~mm}$.

\section{Discussion}

The Tropical Andes, including parts of Venezuela, Colombia, Ecuador, Peru, Bolivia and Argentina, is the biologically richest and most diverse of Earth's biodiversity hotspots areas, 
mainly for vertebrates and plants (Myers et al. 2000). Although that area retains about 25\% of its primary vegetation, is believed to contain, at least, 20,000 known plant endemics and probably thousands of species remain to be discovered there (Myers et al. 2000). Because many insects are associated with plants, the extreme plant endemism of the Tropical Andes suggests a similar high level of insect diversity and endemism (Larsen et al. 2011).

The high altitudes of the Andes (above 3000 masl) include the most endangered and vulnerable ecosystem in South America and it is one of the three areas where the largest changes in fauna are predicted as a result of climate change (e.g. Lawler et al. 2009, Larsen et al. 2011).

Montoya et al. (2012) found that the Colombian Andes has the highest diversity and number of endemic species of Syrphidae. Species in the Neotropical genera Macrometopia Philippi, 1865, Meropidia, Talahua Fluke, 1943 and Tuberculanostoma Fluke, 1943 were in their study found to be restricted to the high altitudes above 3000 masl of the Colombian Andes.

Therefore, these taxa might be considered focus groups in future conservation projects due to the predictions of the impact of the climate change. The species described here also occur at altitudes between 2000-3000 masl and are thus important additions to the knowledge of the biodiversity of the high Andes flower fly fauna.

Of the five Meropidia species now known for the science, the male sex is still only known for $M$. neurostigma. The hitherto described Meropidia species are distributed in Bolivia, Colombia and Ecuador.

\section{Acknowledgments}

We thank Nigel Wyatt (Natural History Museum, London) for kindly providing pictures of Meropidia nigropilosa Thompson. The first author thanks CNPq, Science Without Borders Program, Brazil, for financial support (proc. 201536/2012-9).

\section{References}

Carvalho Filho F da S, Esposito MC (2009) A review of the flower fly genus Ornidia Lepeletier \& Serville (Diptera: Syrphidae) with the description of a new species from Brazil. 2014: 59-64. http://www.mapress.com/zootaxa/2009/f/z02014p064f.pdf

Gutierrez C, Carrejo NS, Ruiz C (2005) Listado de géneros de Syrphidae (Diptera: Syrphoidea) de Colombia. Biota Colombiana 6: 173-180. http://www.redalyc.org/articulo. oa?id $=49160203$

Hippa H, Thompson FC (1983) Meropidia, a new genus of flower flies (Diptera: Syrphidae) from South America. Papéis Avulsos de Zoologia 35(9): 109-115. http://si-pddr.si.edu/ dspace/handle/10088/18911

Larsen TH, Escobar F, Armbrecht I (2011) Insects of the Tropical Andes: Diversity Patterns, Processes and Global Change. In: Herzog SK, Martinez R, Jørgensen PM, Tiessen H (Eds) 
Climate Change and Biodiversity in the Tropical Andes. Inter-American Institute of Global Change Research (IAI) and Scientific Committee on Problems of the Environment (SCOPE), 228-244. http://www.iai.int/files/communications/publications/scientific/Climate_Change_and_Biodiversity_in_the_Tropical_Andes/book.pdf

Lawler JL, Shafer SL, White D, Kareiva P, Maurer EP, Blaustein AR, Bartlein PJ (2009) Projected climate-induced faunal change in the Western Hemisphere. Ecology 90: 588-597. doi: 10.1890/08-0823.1

Montoya AL, Pérez SP, Wolff M (2012) The diversity of flower flies (Diptera: Syrphidae) in Colombia and their Neotropical distribution. Neotropical Entomology 41: 46-56. doi: 10.1007/s13744-012-0018-z

Morales MN, Massardo D, Moreira GRP, Thompson FC (2009) A new species of Alipumilio Shannon (Diptera, Syrphidae) found in association with the exudate resin of Schinus terebinthifolius Raddi (Anacardiaceae). Zootaxa 2112: 53-64. http://si-pddr.si.edu/dspace/ bitstream/10088/16773/1/ent_FCT_121.pdf

Myers N, Mittermeier RA, Mittermeier CG, Fonseca GAB da, Kent J (2000) Biodiversity hotspots for conservation priorities. Nature 403: 853-858. doi: 10.1038/35002501

Ricarte A, Marcos-García MA, Hancock EG, Rotheray EG (2012) Revision of the New World genus Quichuana Knab, 1913 (Diptera: Syrphidae), including descriptions of 24 new species. Zoological Journal of the Linnean Society 166: 72-131.

Rotheray GE, Hancock EG, Marcos-García MA (2007) Neotropical Copestylum (Diptera, Syrphidae) breeding in bromeliads (Bromeliaceae) including 22 new species. Zoological Journal of the Linnean Society 150(2): 267-317. doi: 10.1111/j.1096-3642.2007.00288.x

Rotheray GE, Marcos-García MA, Hancock G, Pérez-Bañón C, Maier CT (2009) Neotropical Copestylum (Diptera, Syrphidae) breeding in Agavaceae and Cactaceae including seven new species. Zoological Journal of the Linnean Society 156(4): 697-749. doi: 10.1111/j.10963642.2008.00503.x

Thompson FC (1972) A contribution to a generic revision of the neotropical Milesinae (Diptera, Syrphidae). Arquivos de Zoologia 23(2): 73-215. http://www.revistas.usp.br/azmz/ article/view/11964/13741

Thompson FC (1999) A key to the genera of the flower flies (Diptera: Syrphidae) of the Neotropical region including descriptions of new genera and species and a glossary of taxonomic terms. Contributions on Entomology, International 3(3): 321-378.

Thompson FC (2013) Syrphidae. Systema Dipterorum, Version 1.5. 13354 records. http://www. diptera.org/ [accessed on 5 September 2013]

Thompson FC, Marinoni L (2003) Copestylum circumdatum (Walker) (Diptera: Syrphidae). Redescription of a Neotropical flower fly, with lectotype designations, and new synonyms. Entomological News 114(4): 217-223. http://si-pddr.si.edu/dspace/bitstream/10088/17053/1/ ent_FCT_106.pdf

Thompson FC, Rotheray GE, Zumbado MA (2010) Syrphidae (Flower flies). In: Brown BV, Borkent A, Cumming JM, Wood DM, Woodley NE, Zumabdo MA (Eds) Manual of Central American Diptera. Volume 2, NRC Research Press, Ottawa, Canada, 763-792. 